\title{
VALIDAÇÃO DA VERSÃO ADAPTADA DO PARENT PERCEPTION INVENTORY - PPI EM CRIANÇAS BRASILEIRAS DE 7 A 12 ANOS
}

\author{
VALIDACIÓN DE LA VERSIÓN ADAPTADA DEL PARENT PERCEPTION \\ INVENTORY - PPI PARA NIÑOS BRASILEÑOS DE 7 A 12 AÑOS
}

\author{
VALIDATION OF THE ADAPTED VERSION OF THE PARENT PERCEPTION \\ INVENTORY - PPI FOR BRAZILIAN CHILDREN AGED 7 TO 12 YEARS
}

\author{
Elizangela Moreira Careta GALINDO ${ }^{1}$ \\ Ana Maria Pimenta CARVALHO ${ }^{2}$
}

RESUMO: A temática da criação dos filhos vem sendo muito pesquisada e diversos trabalhos têm dedicado atenção especial aos estilos e práticas parentais. Desde a década de 1930, psicólogos pesquisadores e clínicos têm se preocupado com questões como: Qual a melhor forma de educar os filhos e quais são as conseqüências que podem ser provocadas no desenvolvimento das crianças educadas por diferentes modelos de pais. Considerando a relevância da relação entre pais e filhos no processo de desenvolvimento, este estudo objetivou-se validar, através da análise fatorial confirmatória, uma versão adaptada para crianças brasileiras do Parent Perception Inventory - PPI, para mensurar o comportamento dos pais com relação às práticas educativas na percepção dos filhos. Participaram do estudo 600 crianças de 7 a 12 anos, de ambos os sexos, de escolas públicas e privadas da cidade de Ribeirão Preto-SP que responderam duas versões do PPI, uma para o pai e outra para a mãe, cada uma com 18 itens que avaliam nove classes de comportamento positivo e nove classes de comportamento negativo. Por meio do método de Máxima Verossimilhança pôde-se confirmar o modelo de duas dimensões, fatores positivos e negativos, referentes às práticas adotadas, distribuídos respectivamente nos itens impares e pares da escala para as duas versões. Tais componentes apresentaram Alfa de Cronbach entre 0.67 e 0.81 e se correlacionaram positivamente com os domínios da escala utilizada para testar a validade convergente. Conclui-se que o instrumento é valido e pode ser usado em estudos para identificar o comportamento parental do ponto de vista da criança.

PALAVRAS-CHAVE: Criança. Práticas parentais. Instrumentos psicológicos. Análise fatorial confirmatória.

RESUMEN: La temática de la educación de los hijos viene siendo muy investigada y diversos trabajos han dedicado atención especial a los estilos y prácticas parentales. Desde la década de 1930, psicólogos investigadores y clínicos se han preocupado por cuestiones

1 Universidade de São Paulo (USP), Ribeirão Preto - SP - Brasil. Doutoranda em ciências. Faculdade de Medicina de Ribeirão Preto da Universidade de São Paulo (FMRP-USP) - Escola de Enfermagem de Ribeirão Preto - EERP/USP. ORCID: <https://orcid.org/0000-0003-3173-9150>. E-mail: lisamcgalindo@ gmail.com ${ }^{2}$ Universidade de São Paulo (USP), Ribeirão Preto - SP - Brasil. Faculdade de Medicina de Ribeirão Preto da Universidade de São Paulo (FMRP-USP) - Escola de Enfermagem do Departamento de Enfermagem Psiquiátrica e Ciências Humanas - EERP/USP. ORCID: 〈https://orcid.org/0000-0003-2844-3682>. E-mail: anacar7@usp.br 
como: ¿Cuál es la mejor forma de educar a los hijos y cuáles son las consecuencias que pueden ser provocadas en el desarrollo de los niños educados por diferentes modelos de padres? En este estudio se objetivó validar, a través del análisis factorial confirmatorio, una versión adaptada para niños brasileños del Parent Perception Inventory - PPI, para medir el comportamiento de los padres con relación a las prácticas educativas en la percepción de los hijos. En el estudio participaron 600 niños de 7 a 12 años, de ambos los sexos, de escuelas públicas y privadas de la ciudad de Ribeirão Preto (Brasil) que respondieron dos versiones del instrumento, una para el padre y otra para la madre, cada una con 18 puntos que evalúan nueve clases de comportamiento positivo y nueve clases de comportamiento negativo. Por medio del método de Máxima Verosimilitud se pudo confirmar el modelo de dos dimensiones, factores positivos y negativos, referentes a las prácticas adoptadas, distribuidos respectivamente en los puntos impares y pares de la escala para las dos versiones. Tales componentes presentaron Alfa de Cronbach entre 0.67 y 0.81 y se correlacionaron positivamente con los dominios de la escala utilizada para probar la validez convergente. Se concluye que el instrumento es válido y puede ser utilizado en estudios para identificar el comportamiento parental desde el punto de vista del niño.

PALABRAS CLAVE: Niño. Prácticas parentales. Instrumentos psicológicos. Análisis factorial confirmatorio.

ABSTRACT: The theme of raising a child has received attention of many researchers and several papers have devoted particular attention to parental styles and practices. Since the 1930s, psychology researchers, and clinicians have been concerned with issues such as: What is the best way to educate children and what are the consequences that can be caused in their development when educated by different models of parents. Considering the relevance of the relationship between parents and children in the development process, this study aimed to validate, through the confirmatory factorial analysis, a version adapted of the Parent Perception Inventory PPI for Brazilian children to measure children's perceptions of parents' behavior. The study included 600 children aged from 7 to 12 years, both boys and girls, from public and private schools in the city of Ribeirão Preto-SP, who answered two versions of the PPI, one for the father and one for the mother, each with 18 items which evaluate nine classes of positive behavior and nine classes of negative behavior. By means of the Maximum Likelihood method, the two-dimensional model, positive and negative, could beconfirmed, referring to the adopted practices, distributed respectively in the odd and even items of the scale for the two versions. These components presented Cronbach's alpha between 0.67 and 0.81 and correlated positively with the domains of the scale used to test the convergent validity. We conclude that the instrument is valid and can be used in studies to identify parental behavior from the child's point of view.

KEYWORDS: Child. Parent practices. Psychological instruments. Confirmatory factorial analysis.

\section{Introdução}

Desde a década de 1930, psicólogos pesquisadores e clínicos têm se preocupado com questões como: Qual a melhor forma de educar os filhos e quais são as conseqüências que 
podem ser provocadas no desenvolvimento das crianças educadas por diferentes modelos de pais (DARLING \& STEINBERG, 1993).

Ao longo do século XX, no mundo ocidental, a atenção ao cuidado com a criança foi uma constante, mas as formas de lidar e educar e os responsáveis por esses aspectos se modificaram no decorrer do tempo.

A partir da década de 50 os pais vieram adotando uma forma menos punitiva, com a psicologia e a psicanálise influenciando as crenças e atitudes sobre a infância com o intuito de proporcionar uma infância mais feliz.

Diversos trabalhos têm dedicado atenção especial aos estilos e práticas parentais. (ALVARENGA \& PICCININI, 2001; CARVALHO E GOMIDE, 2005; COSTA, TEIXEIRA E GOMES, 2000; WEBER et al., 2004).

Os estilos parentais representam o contexto em que os pais influenciam seus filhos através de suas práticas, de acordo com suas crenças e valores. São tendências relativamente estáveis através das quais os pais reagem com uma conduta (ou prática) dirigida à criança. Desta forma, o estilo e a prática educativa estão normalmente associados, uma vez que o conjunto de práticas educativas utilizadas pelos pais na interação com os filhos formará o estilo parental, ou seja, as práticas educativas, combinadas de diferentes formas, resultam em estilos parentais diversos.

Baumrind (1966) destacou três modelos de estilos parentais adotados na educação dos filhos: o estilo autorizativo, autoritário e permissivo. Tais estilos trouxeram contribuições para o entendimento do processo de socialização da criança.

Seus estudos mostraram que as crianças educadas por diferentes estilos de comportamento dos pais diferiam no grau de competência social. Além de os estilos parentais influenciarem em diversos aspectos no desenvolvimento dos filhos, podem estar determinando o estilo parental que os filhos vão adotar futuramente, havendo uma transmissão intergeracional de estilos parentais.

Maccoby e Martin (1983) reorganizaram os protótipos de Baumrind em duas dimensões: exigência e responsividade. As características de cada estilo puderam ser sistematizadas através destas duas dimensões. Os pais autoritários eram considerados exigentes e não responsivos (as exigências deles estão em desequilíbrio com a aceitação das exigências dos filhos). Os pais autoritativos eram exigentes e responsivos (há uma reciprocidade, os filhos devem responder às exigências dos pais, mas estes também aceitam a responsabilidade de responderem, o quanto possível, aos pontos de vista e razoáveis exigências dos filhos. Os pais permissivos são não exigentes e nem responsivos (tendem a 
orientar-se pela esquiva das inconveniências, o que os faz responder a pedidos imediatos das crianças apenas de forma a findá-los).

Em 1983, Harzard, Christensen e Margolin, desenvolveram o Parent Perception Inventory (PPI) para avaliar a percepção que a criança tem de seus pais através de dezoito classes de comportamento parental, positivos (reforço positivo, consolo, tempo de conversa, envolvimento na tomada de decisões, tempo juntos, avaliação positiva, permitir independência, oferecer assistência, afeição não verbal) e negativos (remoção de privilégio, crítica, ordem, punição física, gritos, ameaças, castigo, irritação e ignorar). O instrumento possui 2 versões, uma versão para pais e outra para a criança onde respondem sobre a percepção das práticas educativas parentais.

No Brasil, Teixeira, Bardagi e Gomes (2004) apresentaram um estudo de um instrumento refinado que avalia responsividade e exigência percebidos na adolescência. Indicado para adolescentes entre 14 e 19 anos, o instrumento apresenta 24 itens, 12 relacionados à exigência e 12 à responsividade. Neste estudo as mães foram percebidas como mais responsivas e exigentes do que os pais, tanto pelas filhas quanto pelos filhos. Esses resultados podem estar confirmando uma maior proximidade da mãe com os filhos, apontada em estudos anteriores, nos quais as mães aparecem como bastante envolvidas na educação e desenvolvimento dos filhos, suplantando em muitos aspectos a participação paterna.

Gomide (2006) selecionou em seu modelo teórico sete práticas educativas que compõem o Estilo Parental. Seus estudos resultaram na elaboração do Inventário de Estilos Parentais - IEP, um instrumento destinado à adolescentes de 13 a 16 anos e composto por 42 itens que avalia os estilos parentais através de 7 práticas educativas, cinco relacionadas ao desenvolvimento de comportamento antissociais (negligência, punição inconsistente, disciplina relaxada, monitoria negativa e abuso físico) e duas relacionadas ao comportamento pró-sociais (comportamento moral e monitoria positiva). O IEP pode ser utilizado como medida de pré e pós testes, coleta de dados, para correlações com outros instrumentos, avaliação de nível de risco etc. Ressalta-se que ele é o primeiro instrumento brasileiro validado que possibilita a avaliação de sujeitos de risco, sendo, portanto, de grande demanda por parte de pesquisadores e profissionais da área.

Pasquali e Cols. (2012) elaboraram uma versão reduzida de um instrumento brasileiro cujo objetivo foi proporcionar um levantamento da percepção dos filhos acerca do comportamento e atitude de seus pais, o Questionário de Percepção dos pais - QPP. O instrumento é indicado para crianças e adolescentes na faixa etária de 10 a 16 anos e composto por 20 itens que se distribuem nas dimensões de responsividade, traduzidos por 
comportamentos como comunicação aberta, promoção de apoio e demonstração de afeto e harmonia na relação entre pais e filhos; e exigência, que enfocou aspectos de monitoração de comportamento, exigência e controle, refletindo a percepção dos pais como figura de autoridade. $\mathrm{O}$ objetivo foi se apropriar de uma versão mais prática e funcional.

As práticas educativas parentais, por sua vez, correspondem a comportamentos definidos por conteúdos específicos. São estratégias usadas para suprimir comportamentos considerados inadequados ou incentivar a ocorrência de comportamentos desejados.

A paternidade é um processo evolutivo ao longo da vida da criança e ao atuarem como agentes de socialização dos filhos, os pais utilizam diversas estratégias e técnicas para orientar seus comportamentos (DARLING; STEINBERG, 1993; GOMIDE et al., 2005; HOFFMAN, 1975).

A seleção dessas práticas está guiada pelas metas, as quais são embasadas pelos valores e estilos parentais (ALVARENGA; PICCININI, 2001; BEM; WAGNER, 2006; CARVALHO; GOMIDE, 2005; KOBARQ,VIEIRA; VIEIRA, 2010; OLIVEIRA et al., 2002; PICCININI et al.,2007; WEBER et al., 2004; WEBER et al.,2006).

É importante conhecer não somente as práticas educativas, como também as repercussões que estas têm na vida da criança (MONTANDON, 2005).

Os estudos sobre as práticas parentais e estilos parentais requereram a elaboração de instrumentos que pudessem mensurá-los. Holden (1995) argumenta que desde o início do século, mais de 100 instrumentos diferentes foram utilizados na avaliação da prática parental com o objetivo de identificar as atitudes que beneficiam o processo de educação da criança.

$\mathrm{O}$ interesse em avaliar as práticas parentais justifica-se por sua associação com o desenvolvimento infantil, podendo ser um fator de proteção ou de risco e estudar crianças mais jovens em idade escolar levou a opção pelo instrumento Parent Perception Inventory (PPI), também pelo fato de faltarem estudos mais detalhados sobre suas propriedades psicométricas além da avaliação da consistência interna (HAZZARD E COLS, 1983; GLASER, 1995).

O presente estudo visa dar continuidade ao processo de adaptação e análise fatorial exploratória do Parent Percetion Inventory - PPI, cujo objetivo é confirmar sua estrutura fatorial de 2 dimensões através da análise fatorial confirmatória.

\section{Método}


Trata-se de um estudo metodológico de abordagem quantitativa. Participaram da amostra 600 crianças com idades entre 7 e 12 anos incompletos, de ambos os sexos, de escolas públicas e privadas da cidade de Ribeirão Preto. Tratou-se de uma amostra por conveniência visto que foi composta pelas crianças que quiseram participar e que foram autorizadas por seus pais. Foi informado sobre a confiabilidade dos dados obtidos e da possibilidade de desistir a qualquer momento sem nenhum prejuízo.

A escolha da faixa etária levou em consideração que a criança de 7 anos já é alfabetizada e consegue responder ao questionário de forma coletiva mantendo a uniformidade na coleta dos dados. O critério 12 anos incompletos foi adotado porque essa faixa delimita o que o Estatuto da Criança e do Adolescente considera como criança. (Art. 2. ${ }^{\circ}$ da lei número 8069 de 13 de julho de 1990 “Considera-se criança, para os efeitos desta Lei, a pessoa até doze anos de idade incompletos, e adolescente aquela entre doze e dezoito anos de idade").

A aplicação foi feita de forma coletiva, lendo-se as instruções até que fosse entendido o conceito e na sequência, foi lida cada sentença para que cada criança anotasse sua resposta. Para isso foi usado um gráfico em forma de pizza onde a criança identificava a resposta conforme sua intensidade. Foi trabalhado em grupos de 10 crianças em cada aplicação para um maior controle do aplicador e garantindo que as respostas fossem fidedignas à opinião de cada um e levaram em média 30 minutos para responder aos questionários.

$\mathrm{Na}$ análise fatorial confirmatória os dados foram analisados por meio do método de Máxima Verossimilhança através das medidas de qualidade de ajuste do modelo (medidas de ajuste absoluto, medidas de ajuste incremental e medidas de ajuste parcimonioso) (HAIR, 2005). A confiabilidade do instrumento foi verificada por meio da estatística alfa de Cronbach.

O presente projeto foi submetido à avaliação do Comitê de Ética em Pesquisa da Escola de Enfermagem de Ribeirão Preto - SP, sendo aprovado em 27 de setembro de 2016 Protocolo CAAE: 57829716.9.00005396. Foi solicitado aos pais seu consentimento para que as crianças participassem da pesquisa, e o assentimento das crianças, obedecendo a Resolução $\mathrm{n}^{\circ} 466 / 2012$ sobre pesquisas que envolvem seres humanos 0560/05

\section{Resultados}

Os participantes se caracterizaram por maior número de participantes do sexo masculino representando $51.3 \%$ dos sujeitos $(n=308)$ e $48.7 \%$ do sexo feminino $(n=292)$. As 
idades estão distribuídas da seguinte forma: 7 anos (113 sujeitos representando $18.83 \%$ da amostra), 8 anos (133 sujeitos representando $22.17 \%$ da amostra), 9 anos (164 sujeitos representando $27.33 \%$ da amostra), 10 anos (107 sujeitos representando $17.83 \%$ da amostra) e 11 anos (83 sujeitos representando $13.83 \%$ da amostra). A esfera escolar mostra que $87 \%$ da amostra são crianças de escolas públicas, representando um n de 522 sujeitos enquanto que $13 \%$ de escolas particulares com n igual a 78 sujeitos. Segundo Critério de classificação Econômica Brasil, representada pelo poder de compra da população e não em classes sociais, $1.3 \%(n=8)$ da amostra estudada são de classe A1; $9 \%(n=54)$ são de classe A2; $11.3 \%(n=68)$ são de classe B1; 32.3\% (n=194) são de classe B2; 29.2\% $(n=175)$ são de classe $C 1 ; 13.7 \%$ $(n=82)$ são de classe $C 2$ e $3.2 \%(n=19)$ são de classe $D$.

Com relação às medidas de confiabilidade e validade, todos os parâmetros estimados dos itens e dos fatores, das duas versões em que a criança avalia a mãe e o pai, apresentaram resultados satisfatórios. Todas as variâncias foram positivas com exceção da covariância entre os dois fatores do instrumento, cujos valores são iguais a -0,0993 para a versão da mãe e 0,1582 para a versão pai, que se explicam pelo fato de um fator ser inverso ao outro, ou seja, são comportamentos contrários, cuja prática educativa parental tem a função de incentivar ou inibir o comportamento da criança.

A confiabilidade do instrumento foi verificada por meio da estatística alfa de Cronbach. O valor geral de alfa é de 0,7559 para a versão mãe e alfa igual a 0,8212 para a versão pai. Na versão em que as crianças avaliam a mãe, o fator 1 (práticas positivas) apresentou alfa de 0.671 com intervalo entre 0,6379 e 0,7041. No fator 2 (práticas negativas), o alfa foi de 0.7438 com intervalo entre 0,7173 e 0,7689 . Na versão do Pai o alfa encontrado foi de 0.8194 para o fator 1 com intervalo de confiança entre 0,8007 e 0,8371 . Para o fator $2 \mathrm{o}$ alfa foi de 0.8174 com intervalo entre 0,7984 e 0,8353 .

Tabela 1 - Coeficientes de confiabilidade geral do instrumento

\begin{tabular}{cccc}
\hline Instrumento & Alfa & Mínimo & Máximo \\
\hline & & & \\
PPI mãe & $\mathbf{0 , 7 5 5 9}$ & 0.7314 & 0,7792 \\
\hline & & & \\
PPI pai & $\mathbf{0 , 8 2 1 2}$ & 0,8033 & 0,8383 \\
\hline
\end{tabular}

Fonte: Produzido pelo Autor 
Tabela 2 - Coeficientes de confiabilidade por fator

\begin{tabular}{ccccc}
\hline \multicolumn{2}{c}{ Instrumento } & Alfa & Mínimo & Máximo \\
\hline $\begin{array}{c}\text { PPI } \\
\text { FATOR 1 }\end{array}$ & mãe & & & \\
\hline PPI & mãe & $\mathbf{0 , 6 7 1 9}$ & 0,6379 & 0,7041 \\
\hline FATOR 2 & & $\mathbf{0 , 7 4 3 8}$ & 0,7173 & 0,7689 \\
\hline PPI & pai & & & \\
FATOR 1 & & $\mathbf{0 , 8 1 9 4}$ & 0,8007 & 0,8371 \\
\hline PPI & pai & & & \\
FATOR 2 & & $\mathbf{0 , 8 1 7 4}$ & 0,7984 & 0,8353 \\
\hline
\end{tabular}

Fonte: Produzido pelo Autor

O presente estudo partiu do princípio que a estrutura do instrumento comporta dois fatores. Dessa forma, procedeu-se à na Análise Fatorial Confirmatória, utilizando-se os indicadores de ajuste capazes de verificar se o modelo fatorial é consistente com as variáveis iniciais para testar a matriz de covariância predita. As medidas de qualidade de ajuste foram calculadas segundo as três formas propostas pela literatura: medidas de ajuste absoluto, medidas de ajuste incremental e medidas de ajuste parcimonioso para cada versão do instrumento (HAIR, 2005). Na versão em que as crianças avaliam a mãe, as medidas de ajuste absoluto, o qual avalia o ajuste geral do modelo, a Raiz do Erro Quadrático Médio de Aproximação (RMSEA) e a Raiz do Resíduo Quadrático Médio (SMSR) tiveram os valores 0.0391 e 0.0444 respectivamente. Com relação ao ajuste parcimonioso, o qual acerta a medida de ajuste e fornece uma comparação entre os modelos, apresentou o índice de ajuste ponderado (NFI) o valor de 0.8415 (um pouco abaixo do valor de referência, mas próximo do ideal) e o índice de ajuste comparativo (CFI) igual a 0.9162. As medidas de ajuste incremental, o qual compara o modelo proposto com o modelo especificado pelo pesquisador, apresentou o índice ajustado de qualidade do ajuste (AGFI) igual a 0.9418 e o índice de Tucker Lewis (TLI) igual a 0.9044 . Na versão em que as crianças avaliam os pais os índices se apresentaram da seguinte forma: RMSEA 0.0412, SRMR 0.0496, AGFI 0.938, TLI 0.9442, NFI 0.9083 e CFI 0.9512. De um modo geral os resultados mostraram um bom ajuste com valores aceitáveis de qualidade. 
Tabela 3 - Valores obtidos das medidas de ajuste

\begin{tabular}{lccc}
\hline Grupo & Medidas & Mães & Pais \\
\hline Ajuste absoluto & RMSEA & 0,0391 & 0,0412 \\
& SRMR & 0,0444 & 0,0496 \\
Ajuste incremental & AGFI & 0,9418 & 0,938 \\
& TLI & 0,9044 & 0,9442 \\
Ajuste parcimonioso & NFI & 0,8415 & 0,9083 \\
& CFI & 0,9162 & 0,9512 \\
\hline
\end{tabular}

Fonte: Produzido pelo Autor

A análise discriminante foi realizada considerando a variável sexo da criança. Os resultados mostraram que só houve diferença com relação ao sexo da criança no que se referia à avaliação do pai, quanto às práticas negativas (fator 2).

Tabela 6 - Valores referentes à validação discriminante quanto ao sexo da criança.

\begin{tabular}{ccc}
\hline & Fator 1 / P valor & Fator 2 / P valor \\
\hline PPI Mãe & 44664,5 / 0,8863 & 48414 / 0,1040 \\
PPI Pai & 43112,5 / 0,3816 & 49464,5 / 0,0339 \\
\hline
\end{tabular}

Fonte: Produzido pelo Autor

\section{Discussão}

A proposta deste estudo foi verificar as propriedades psicométricas do instrumento Parent Perception Inventory - PPI por meio da Análise Fatorial Exploratória, na versão que avalia as práticas educativas parentais, do ponto de vista dos filhos, esperando que se confirmem essas propriedades conforme o instrumento original. Confia-se que o objetivo tenha sido alcançado. Buscou-se ampliar a amostra cujo número de participantes foi estabelecido a partir de cálculo amostral necessário para se efetivar a Análise Fatorial Confirmatória.

A faixa etária escolhida no presente estudo foi de 7 a 12 anos incompletos, para que não fossem abordados adolescentes. Identificou-se que algumas crianças de sete anos, provenientes de escolas públicas, apresentaram maior limitação de compreensão das questões 
apresentando dificuldade em responder de forma coletiva. Alguns autores como Scala et. Al. (2005), atribuem essa dificuldade à alfabetização precária em que se encontram as crianças brasileiras somada ao baixo nível cultural atribuído à essa faixa etária, que impede a compreensão das palavras comuns.

As respostas das crianças ao completarem o PPI, mostraram que os filhos, de um modo geral, percebem seus pais com menos comportamentos negativos que suas mães e as mães também foram consideradas como mais exigentes que os pais. Com relação às práticas positivas da mãe, a maioria das crianças responderam que o comportamento se aplica bastante com exceção dos itens 7 e 13, que se referem às questões relacionadas ao envolvimento na tomada de decisões e comportamentos que permitem a independência dos filhos. Para estes itens as crianças responderam que às vezes a mãe permite o comportamento descrito.

Com relação aos itens que descrevem as práticas negativas da mãe, a maioria das crianças considera que a mãe nunca apresenta o comportamento descrito, com exceção do item 2, que traduz a remoção de privilegio onde são retiradas coisas ou atividades agradáveis para os filhos quando estes se comportam mal, e o item 10 que se refere à repreensão através de gritos. Para esses itens, a maioria das crianças respondeu às vezes e poucas vezes respectivamente. Outro item é o que se refere à frequência com que a mãe dá ordens ao filho (item 6). A maioria das crianças respondeu que as mães apresentam bastante este comportamento, o que as traduzem como muito exigentes.

$\mathrm{Na}$ avaliação dos pais, as práticas positivas se aplicaram bastante pela maioria das crianças em todos os itens do instrumento e com relação às práticas negativas, a maioria das crianças considerou que o comportamento nunca se aplica, com exceção do item 6 , que se refere à frequência que o pai dá ordens e manda no filho, que apareceu bem equilibrada entre nunca, as vezes e bastante nas respostas das crianças.

Os resultados mostraram também que só houve diferença com relação ao sexo da criança no que se referia à avaliação do pai quanto às práticas negativas, e o mesmo resultado foi obtido no QPP com relação ao fator exigência.

Hazzard, Christensen e Margolin (1983), os proponentes do PPI, relatam que as mães são vistas como mais controladoras e exigentes enquanto que os pais mais punitivos. Relatam também que alguns itens no PPI são ações disciplinares e é comum a criança ver isso de forma negativa, mas que podem ser em certas circunstâncias um comportamento parental muito apropriado e essa avaliação negativa que a mãe recebe pode se dar ao fato de estar mais ativa na educação e não ao seu comportamento inapropriado ou patológico. Adicionam ainda 
que estes construtos podem identificar comportamentos parentais problemáticos e medir aspectos mais qualitativos das relações pais e filhos.

Teixeira, Bardagi e Gomes (2004) afirmam que os filhos costumam avaliar seus pais, como menos exigentes e responsivos do que os próprios pais se descrevem, tendendo percebêlos de uma forma mais negativa e essas discrepâncias entre suas percepções tendem a aumentar conforme os filhos vão crescendo e atingindo a adolescência.

A validação do construto foi feita através da análise fatorial confirmatória partindo do pressuposto que o instrumento se mantém em duas dimensões. As dimensões são os fatores positivos e negativos referente às práticas adotadas pelos pais e percebidas pelos filhos, distribuídos respectivamente nos itens pares e ímpares da escala.

A confiabilidade do instrumento foi avaliada por meio da análise da consistência interna de seus itens constatando um valor satisfatório para a amostra estudada. $\mathrm{O}$ instrumento mostrou medidas descritivas e propriedades psicométricas adequadas dentro do que é preconizado. Nesta etapa da validação é importante que se mostre a precisão e a estabilidade do instrumento, ou seja, se ele é estável ao longo do tempo e o quanto ele é capaz de medir o construto. Para isso existem vários métodos e optou-se por utilizar o alfa de Cronbach por ser um dos mais usados apesar de algumas críticas que vem sofrendo. Devido ao alfa usar o número de itens para fazer o seu cálculo, quando o instrumento é grande, beneficia-se apresentando um valor maior e em contrapartida apresenta um valor menor quando o instrumento é considerado pequeno ou com poucos itens. $\mathrm{O}$ valor de alfa geral nas duas versões do instrumento se encontra dentro do intervalo de confiança proposto pela literatura e se aproxima bem dos valores encontrados na validação dos outros instrumentos brasileiros que medem o mesmo construto, o que o torna confiável e apropriado para o uso.

Os estudos realizados o PPI em sua versão original, não foram além da análise exploratória e avaliação da confiabilidade.

Outro fator importante a ser considerado é a facilidade e praticidade que o IPP tem com relação à quantidade de itens que compõem o instrumento, apresentando-se como uma escala breve e de fácil aplicação.

Com relação à qualidade de ajuste do IPP, de um modo geral, os resultados apresentaram valores aceitáveis de qualidade. Embora o valor de ajuste parcimonioso não tenha atingido o valor mínimo proposto na literatura, está bem próximo do valor ideal. Os valores supracitados demonstram que a qualidade de ajuste do modelo proposto poderia ser melhorada se fossem realizadas algumas alterações. Mesmo com o modelo proposto não alcançando todos os valores de corte propostos na literatura, como no caso do ajuste 
parcimonioso NFI (ajuste ponderado), optou-se por não modificar o modelo visando não alterar o modelo teórico proposto inicialmente, pois segundo Marsh et al. (1999), os valores de corte dos índices de ajustamento são linhas orientadoras gerais e não necessariamente regras definitivas. Os demais índices estão de acordo com os valores de corte indicados na literatura evidenciando a adequação geral do modelo em dois fatores e a validade fatorial confirmatória satisfatória.

O presente estudo revelou que duas dimensões são suficientes para mensurar as práticas educativas parentais nesta população, no entanto, a partir dele, não tem a pretensão de oferecer um instrumento que substitua os existentes, mas tornar disponível um que tenha sido construído e validado para o uso com crianças. Dessa forma, resgatar um instrumento de mais de 30 anos pareceria sem sentido, não fosse a adequação de seus parâmetros psicométricos, sua brevidade e facilidade em responder. Além disso, trata-se de um instrumento de uso público, que poderá contribuir com as práticas profissionais daqueles que tem interesse em estudar a relação entre pais e filhos.

\section{Considerações finais}

Foram identificadas algumas limitações com relação à composição da amostra devido à grande negativa por parte dos pais em autorizarem os filhos a participarem do estudo. Outra limitação foi o acesso às escolas particulares que dificultaram a abordagem de seus alunos, justificando-se pelo fato dos pais não gostarem deste tipo de atividade na escola. Algumas crianças menores, provenientes de escolas públicas, não conseguiram responder ao instrumento dentro da metodologia proposta trazendo também uma limitação. Estudos como este requerem que se consiga atingir representatividade da população em que estão sendo conduzidos.

O conteúdo de alguns itens que ainda trouxeram dificuldades para a compreensão das crianças merece atenção em estudos futuros e mesmo reformulação.

O presente estudo possibilitou confirmar as propriedades psicométricas do Inventário de Percepção Parental - IPP e com os resultados aqui obtidos conclui-se que o instrumento é útil, e uma vez estabelecido que ele atende aos objetivos propostos, ou seja, avalia as práticas educativas parentais do ponto de vista do filho, pode-se afirmar que permite o rastreamento de práticas inadequados e indicadoras de risco que possam vir a prejudicar o desenvolvimento infantil. O IPP é válido e fidedigno e pode ser incluído para identificar o comportamento parental problemático do ponto de vista da criança e medir aspectos mais qualitativos nas 
relações pais e filhos. Contudo, deve-se alertar que a amostra utilizada não pode ser considerada representativa da população brasileira o que sugere a necessidade de futuras pesquisas com amostras mais abrangentes e representativas. Outros estudos são necessários para estabelecer-se, por exemplo, valores normativos e padronização, verificando suas propriedades psicométricas em outros contextos, visto que os estudos de validade são constantes e como destaca Urbina (2007), os profissionais da avaliação psicológica devem constantemente se preocupar em buscar evidências de validade para as medidas propostas.

Intervenções em famílias disfuncionais poderão contar com o suporte de um instrumento que auxilie a avaliar sua eficácia, contribuindo para o aprimoramento de trabalhos na área de promoção de saúde mental. Com isso, espera-se que o presente estudo contribua com novas pesquisas e com abordagens que proponham a utilização de instrumentos em diferentes populações. Outros estudos são necessários para estabelecer o poder de discriminar entre grupos de sujeitos e avaliar sua validade preditiva, além da verificação de suas propriedades psicométricas em outros contextos culturais. A despeito de ainda não se poder oferecer dados normativos, é importante ressaltar que o instrumento está pronto para uso em pesquisa e pode auxiliar em avaliações de intervenções junto a famílias.

AGRADECIMENTO: A Jonas Bodini Alonso, estatístico da Escola de Enfermagem de Ribeirão Preto EERP-USP pela consultoria na análise dos dados.

\section{REFERÊNCIAS}

ALVARENGA, P.; PICCININI, C. Práticas educativas maternas e problemas de comportamento em pré-escolares. Psicologia: Reflexão e Crítica, Porto Alegre, v. 14, n. 3, p. 449-460, 2001. Disponível em: http://www.scielo.br/pdf/prc/v14n3/7832.pdf. Acesso em: 05 fev. 2018

BAURIND, D. Effects of authoritative control of child behavior. Child Development, v. 37 , n. 4, p. 887-907, 1966. Disponível em: Acesso em: 5 fev. 2018.

BENETI, S. P. C.; BALBINOTI, M. A. A. Elaboração e estudo de propriedades psicométricas do Inventário de Práticas Parentais, Psico-USF, v. 8, n. 2, p. 103-113, jul./dez. 2003

BERNS, L. A. de.; WAGNER, A. Reflexões sobre a construção da parentalidade e o uso de estratégias educativas em famílias de baixo nível socioeconômico. Psicologia em Estudo, Maringá, v. 11, n. 1, abr. 2006.

CARVALHO, M. C. N.; GOMIDE, P. I. C. Práticas educativas parentais em famílias de adolescentes em conflito com a lei. Estudos de Psicologia, Campinas, v. 22, n. 3, p. 263-274, 2005 
CECCONELLO A. M.; KOLLER S. H. Competência social e empatia: um estudo sobre resiliência com crianças em situação de pobreza. Estudos de Psicologia. 2013

COSTA, F. T. da.; TEIXEIRA, M. A. P.; GOMES, W. B. Responsividade e exigência: duas escalas para avaliar estilos parentais. Psicologia Reflexão e Crítica, v. 13, n. 3, p. 465-473, 2000 .

DANCEY, C.; REIDY, J. Estatística Sem Matemática para Psicologia: usando SPSS para Windows. Artmed, Porto Alegre, 2006.

DARLING, N.; STEINBERG, L. Parenting style as context: an integrative model. Psychological Bulletin, n. 113, 487-496, 1993.

GOMIDE, P. I. C. IEP Inventário de estilos parentais: modelo teórico: manual de aplicação, apuração e interpretação. Petrópolis, Rio de Janeiro: Vozes, 2006.

HAZZARD, A.; CHRISTENSEN, A.; MARGOLIN, G. Children's perceptions of parental behavior. Journal of Abnormal Child Psychology, v. 11. p. 49-60, 1983.

HOFFMAN, M. L. Moral internalization, parental Power and the nature of parent-child interation. Developmental Psychology, v. 11, n. 2, p. 228-239, 1975.

KOBARG, A. P. R.; VIEIRA, V.; VIEIRA, M. L. Validação da Escala de Lembranças sobre Práticas Parentais (Embu). Avaliação Psicológica, Porto Alegre, v. 9, n. 1, 2010.

MACCOBY, E.; MARTIN, J. Socialization in the context of the family: parentchild interaction. In: HETHERINGTON, E. M. (Org.). Handbook of child psychology, v. 4. Socialization, personality, and social development. 4 ed. New York: Wiley, 1983. p. 1-101.

OLIVEIRA, E. A. et al. Estilos parentais autoritário e democrático-recíproco intergeracionais, conflito conjugal e comportamentos de externalização e internalização. Psicologia Reflexão e Crítica, v. 15, p. 1-11, 2002.

PASQUALI, L.; GOUVEIA, V. V.; SANTOS, W. S.; FONSECA, P. N.; ANDRADE, J. M.; LIMA, T. J. S. Questionário de Percepção dos Pais: evidências de uma medida de estilos parentais. Paidéia, Ribeirão preto, v. 22, n. 52, p. 155-164. DOI: 10.1590/S0103863X2012000200002. 2012.

PICCININI, C. A. et al. Práticas educativas de pais e mães de crianças aos 18 meses de idade. Psicologia: Teoria e Pesquisa, v. 23, n. 4, p. 369-378, 2007

SANTOS, C. Estatística Descritiva: mnual de auto-aprendizagem. 2 Ed. Lisboa: Edições Silabo, 2007.

WEBER, L. N. D. et al. Continuidade dos estilos parentais através das gerações-transmissão intergeracional de estilos parentais. Paidéia, Ribeirão Preto, v. 16, n. 35, p. 407-414, 2006.

\section{Como referenciar este artigo}


GALINDO, Elizangela.; CARVALHO, Ana Maria Pimenta. Validação da versão adaptada do Inventário de Práticas Parentais - IPP / Parent Perception Inventory em Crianças brasileiras de 7 a 12 anos. Doxa: Rev. Bras. Psico. e Educ., Araraquara, v. 20, n. 2, p. 164-178, jul./dez., 2018. e-ISSN: 2594-8385. DOI: 10.30715/doxa.v20i2.11602

Submetido em: $27 / 07 / 2018$

Aprovado em: 25/10/2018 